\title{
A Case of Tracheal Amyloidosis Admitted with Rectus Abdominis Muscle Hematoma \\ SA Baris ${ }^{1}, \mathrm{~N}_{\text {Turhan }}{ }^{2}, \mathrm{~K}$ Yildiz ${ }^{3}$, \\ $\mathrm{S}_{\text {Gumustas }}{ }^{4}, \mathrm{~T}$ Onyilmaz ${ }^{5}$, I Basyigit ${ }^{1}, \mathrm{H}$ Boyaci $^{1}$
}

\begin{abstract}
Tracheobronchial amyloidosis is often misdiagnosed as chronic airway diseases in relation to the symptoms of chronic cough and dyspnea. Here, we presented a case admitted to emergency department with severe cough and stomachache and diagnosed with rectus abdominis muscle hematoma due to chronic cough. The case had a history of asthma and no clinical improvement despite the use of inhaled drugs nearly five years. He was diagnosed with tracheobronhial amyloidosis after bronchoscopic and histopathologic evaluation. This case presented to remind the tracheal pathologies in differential diagnosis of chronic cough and emphasize the importance of bronchoscopic evaluation.
\end{abstract}

Keywords: Amyloidosis, cough, hematoma, trachea

From: ${ }^{1}$ Kocaeli University School of Medicine, Department of Pulmonary Diseases, Kocaeli, Turkey ${ }^{2}$ İmit Seka Government Hospital, Department of Pulmonary Diseases, Kocaeli, Turkey ${ }^{3}$ Kocaeli University School of Medicine, Department of Pathology, Kocaeli, Turkey ${ }^{4}$ Kocaeli University School of Medicine, Department of Radiology, Kocaeli, Turkey, ${ }^{5}$ Mardin Government Hospital, Department of Pulmonary Diseases, Mardin, Turkey.

Correspondence: Dr SA Baris, Kocaeli University School of Medicine, Department of Pulmonary Diseases, Umuttepe, Kocaeli, Turkey. E-mail: serapargum2002@yahoo.com 


\section{INTRODUCTION}

Amyloidosis is a local or systemic disease characterized by deposition of abnormal fibrillar and insoluble protein material in the tissues or organs (1). Accumulation of this abnormal protein leads to organ dysfunction (2). Systemic amyloidosis often involves lung parenchyma and the pleura. Localized respiratory tract amyloidosis mostly affects the trachea and the bronchi (3). Here, we presented a case of tracheal amyloidosis admitted with rectus abdominis muscle hematoma due to severe cough episodes that previously misdiagnosed as asthma.

\section{CASE REPORT}

Fifty three years old man admitted to emergency department with the complaints of severe cough and stomachache. He had chronic non-productive cough, diagnosis of asthma and inhaler therapy use nearly five years. He had no clinical improvements despite the inhaler therapy. He had histories of hypertension and benign prostate hypertrophy. He was a currentsmoker with a 55 pack-years of smoking history. His respiratory system examination was normal while there was a swelling approximately $15 \mathrm{~cm}$ in size on right abdominal wall. Laboratory findings were as follows; WBC: 8880 /UL, Hb: 14,9 g/dL, Htc: 45,8\%, C reactive protein $(\mathrm{CRP}): 0,76 \mathrm{mg} / \mathrm{dl}$, erythrocytes sedimentation rate $(\mathrm{ESR}): 30 \mathrm{~mm} / \mathrm{h}$, and biochemical parameters were within the normal range. There was an enlargement of superior mediastinum, narrowing of the trachea and pleural thickening of left hemithorax in chest x-ray. There were bilateral miliary nodules, hilar and mediastinal calcified lymph nodes, pleural thickening and localized thickness on middle part of the right side of tracheal wall in thorax CT (Figure1a,b). 


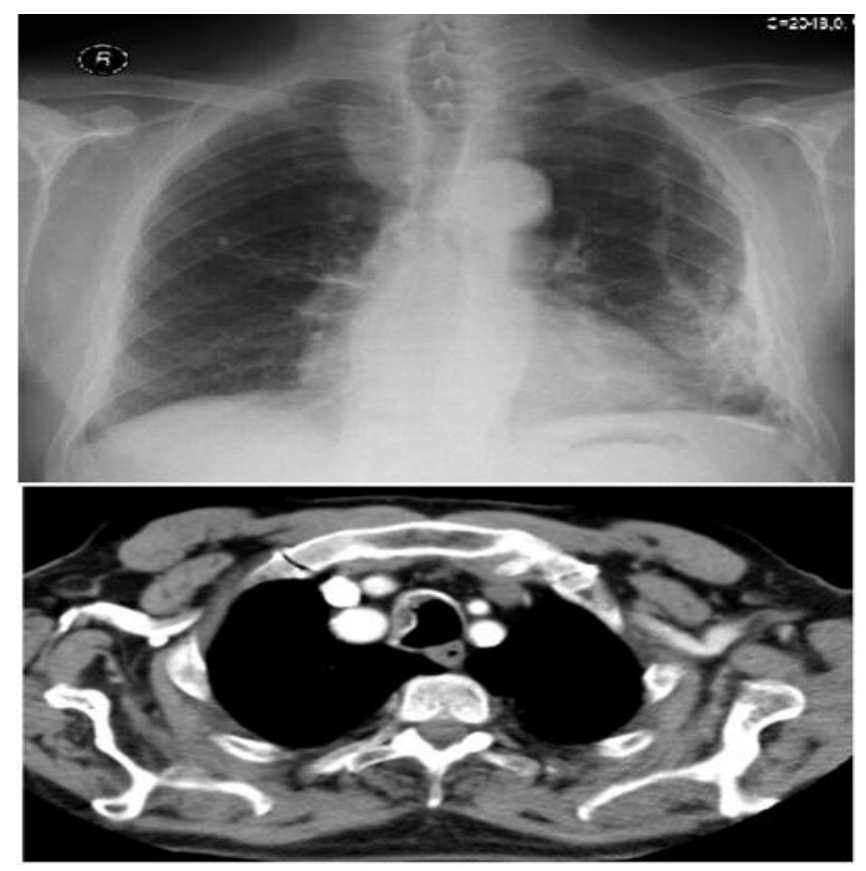

Fig. 1a, b: Enlargement of superior mediastinum in chest x-ray and localized thickness on middle part of the right side of tracheal wall in thorax CT

There was an $18 \times 7 \times 5 \mathrm{~cm}$ sized heterogeneous density consisted with hematoma in the right rectus abdominis muscle in abdominal CT. He was consulted with the department of General Surgery and clinical follow-up was recommended for muscle hematoma.

Fiberoptic bronchoscopy revealed a polypoid lesion in the middle part of the right lateral wall of trachea (Figure-2).

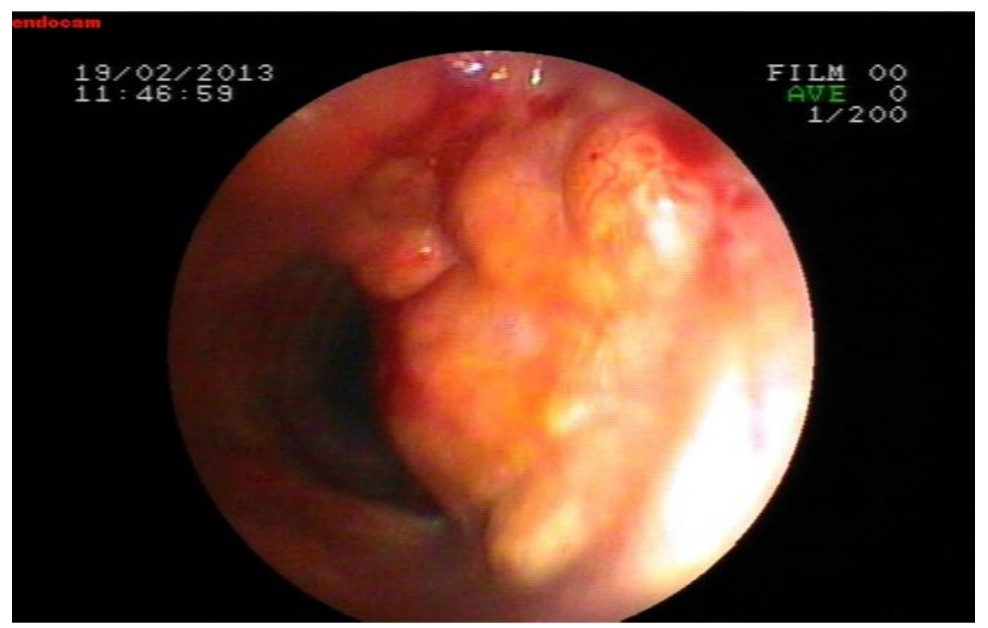


Fig.2: Fiberoptic bronchoscopy revealed a polypoid lesion in the middle part of the right lateral wall of trachea

The results of smear and cytology of the bronchial lavage fluid were negative for acidfast organisms (AFB) and malignant cells. The nonspecific and mycobacterium cultures were negative. The histopathological examination of the biopsy specimens stained positive with PAS and Congo red (Figure 3a, 3b). There was a positive reaction for the AA protein in the immunohistochemical evaluation. According to these results, patient diagnosed with secondary amyloidosis.

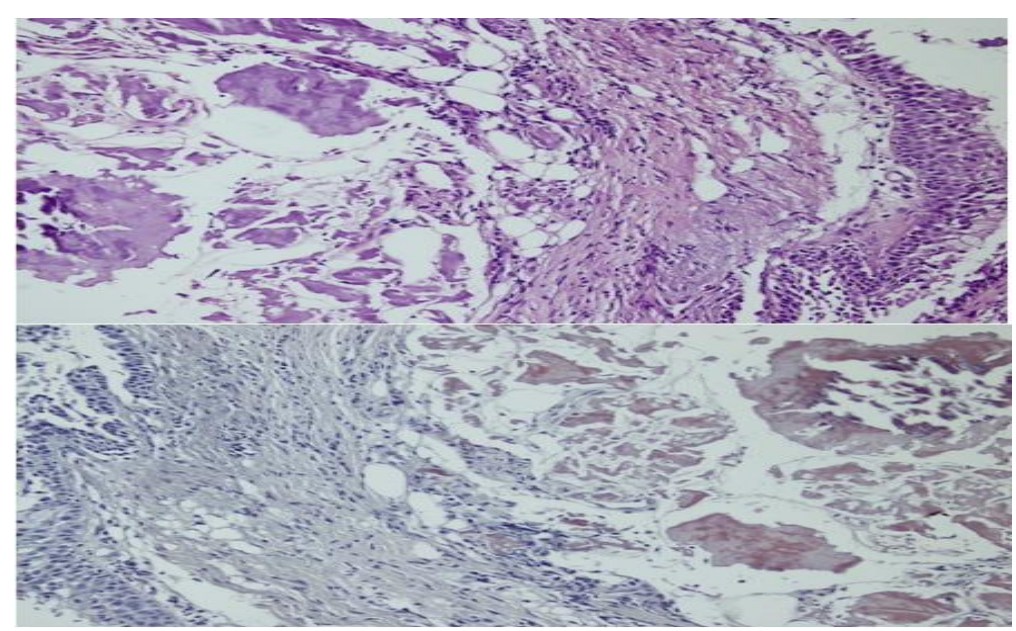

Figure 3a,b: a-The accumulation of eosinophilic amorphous material under respiratory mucosa showing squamous metaplasia (Hematoxylin - eosin x 200).

b- A positive reaction with Congo red staining (x200).

Detailed systemic examination was planned for differentiating the secondary amyloidosis and rule out systemic involvement. He was evaluated by a multi-disciplinary team including the departments of Pulmonary Diseases, Hematology, Rheumatology, Nephrology, Gastroenterology, Infectious Diseases, Urology and Cardiology. Protein electrophoresis and immunoglobulin levels were normal. Echocardiography was normal. The liver and kidney sizes were in normal limits and there was no abnormal finding except hematoma in abdomen CT. Urine immune electrophoresis was normal. There was no supporting finding of multiple myeloma. He also was assessed for chronic infections; hepatic 
markers, anti HIV, brucella and rose bengal test results were negative. He had a history of benign prostate hypertrophia and he was evaluated for excluding the chronic prostatitis. The level of prostate specific antigen, urinary ultrasound and uroflowmetry were also normal. Open lung biopsy was recommended for the diagnosis of parenchymal lung nodules but the patient did not accept the invasive approach. He was referred to a specialized center for endobronchial therapy with the aim of reducing symptoms and improving the quality of life.

\section{DISCUSSION}

Amyloidosis is characterized by abnormal deposition of protein material and its etiology still remains unclear. The major types are AL (immunoglobulin light chain) and AA (amyloidassociated). AL is derived from plasma cells and contains kappa or lambda immunoglobulin light chains; it is associated with primary systemic amyloidosis, myeloma-associated amyloidosis. AA is an immunoglobulin protein synthesized by liver; this protein is deposited in secondary amyloidosis (4). There was a positive reaction of AA protein in the immunohistochemical evaluation of our case and he diagnosed with secondary amyloidosis.

Amyloidosis can be systemic or localized according to the clinical involvement. Three forms of localized amyloidosis were seen in respiratory system;1) nodular opacities, 2) diffuse opacities, 3) tracheobronchial disease (5). The deposition of amyloid material as submucosal plaques and/or polypoid tumors is characteristic in tracheobronchial amyloidosis (6). It is recommended that further investigations should be planned for excluding systemic involvement after the diagnosis of amyloidosis (4). Our case was diagnosed with localized tracheal amyloidosis after the rule out systemic involvement by physical, clinical, chemical and radiological examinations. 
Tracheobronchial amyloidosis is frequent in men and usually occurs in the fifth or sixth decade (5). Our case is 53 years old men and it is consistent with the literature. The symptoms are usually nonspecific. The amount and the distribution of amyloid are decisive in the occurrence of clinical signs. The cases with tracheobronchial amyloidosis present cough, dyspnea or hemoptysis as may be asymptomatic (1). Hoarseness is also one of the admission symptoms. The symptoms are usually nonspecific, mimicking common respiratory conditions such as bronchial asthma $(6,7)$. Also it can misdiagnose as refractory asthma due to uncontrolled symptoms despite of the inhaler use (7). As similar, our case had history of inhaler use for asthma and he had uncontrolled respiratory symptoms. Cough and stomachache were the prominent symptoms while he admitted to emergency department. There was a rectus abdominis muscle hematoma related to rupture of epigastric arteries of the rectus abdominis muscle in abdominal CT. Nearly one third of the rectus muscle hematomas occur due to cough (8). Muscle hematoma is a complication of cough and it is important to determine the cause of persistent respiratory symptoms. It is suggested that the persistent respiratory symptoms of this case might be explained by the misdiagnosed as asthma and tracheal involvement of amyloidosis.

Computed tomography (CT) is the best non-invasive method for evaluation of tracheobronchial lesions (9). The image of tracheobronchial amyloidosis on CT appears as circumferential wall thickening with calcifications as well as luminal narrowing at various levels of the tracheobronchial tree, as was seen in our patient (4). However, the diseases related with tracheobronchial involvement such as tracheobronchial tuberculosis or polychondritis should be considered for differantial diagnosis (9-11).

Tissue biopsy is necessary for definitive diagnosis of amyloidosis. The avidity for Congo red and metachromatic birefringence under unidirectional polarized light remain the gold standard. Once the initial diagnosis has been made, the amyloid subtype must be 
identified and systemic organ involvement must be evaluated (12). The histopathological examination of the biopsy specimens stained positive with PAS and Congo red in our case. There was a positive reaction for the AA protein in the immunohistochemical evaluation.

Most of the cases with tracheobronchial amyloidosis need therapeutic interventions for the control of progressive symptoms (13). It is possible to control of the symptoms and improve the functional status by the techniques such as surgical excision, argon coagulation, cryotherapy, radiotherapy, laser ablation and stent $(13,14)$. Also our case was symptomatic and he was referred to a specialized center of endobronchial therapy for reduce symptoms and improving quality of life.

In conclusion, tracheobronchial amyloidosis is an uncommon disease which is generally misdiagnosed as airway diseases related to symptoms of chronic cough and dyspnea. Tracheal pathologies should be considered in differential diagnosis of chronic cough and bronchoscopic evaluation should be planned in uncontrolled asthmatic patients with an atypical clinical presentation. 


\section{REFERENCES}

1. Capizzi SA, Betancourt E, Prakash UB. Tracheobronchial amyloidosis. Mayo Clin Proc 2000; 75: 1148-52.

2. Sommer P, Kumar G, Lipchik RJ, Patel JJ. Tracheobronchial amyloidosis managed with multimodality therapies. Ther Adv Respir Dis 2014; 8: 48-52.

3. Xu L, Cai BQ, Zhong X, Zhu YJ. Respiratory manifestations in amyloidosis. Chin Med J 2005; 118: 2027-33.

4. Wang Q, Chen H, Wang S. Laryngo-tracheobronchial amyloidosis: a case report and review of literature. Int J Clin Exp Pathol 2014; 7: 7088-93.

5. Berk JL, O’Regan A, Skinner M. Pulmonary and tracheobronchial amyloidosis. Semin Respir Crit Care Med 2002; 2: 155-65.

6. Serraj M, Kamaoui I, Znati K, Kouara S, Sahnoune F, Amara B, et al. Pseudotumoral tracheobronchial amyloidosis mimicking asthma: a case report. J Med Case Rep 2012; 6: 40 .

7. Ozyigit LP, Kiyan E, Okumus G, Yilmazbayhan D. Isolated laryngotracheal amyloidosis presenting as a refractory asthma and longstanding hoarseness. $\mathbf{J}$ Asthma 2009; 46: 314-7.

8. Sheth HS, Kumar R, DiNella J, Janov C, Kaldas H, Smith RE. Evaluation of Risk Factors for Rectus Sheath Hematoma. Clin Appl Thromb Hemost 2014; pii: 1076029614553024. [Epub ahead of print]

9. Acar T, Bayraktaroglu S, Ceylan N, Savas R. Computed tomography findings of tracheobronchial system diseases: a pictorial essay. Jpn J Radiol 2015; 33: 51-8.

10. Guo X, Wang C, Wang X, Ma J, Xv L, Luan T et al. Characteristics and risk factor analysis of 410 cases of tracheobronchial tuberculosis. Exp Ther Med 2014; 8: 781-4. 
11. Sharma A, Law A, Bambery P, Sagar V, Wanchu A, Dhir V et al. Relapsing polychondritis: clinical presentations, disease activity and outcomes. Orphanet J Rare Dis 2014; 20: 198.

12. Real de Asúa D, Costa R, Galván JM, Filigheddu MT, Trujillo D, Cadiñanos J. Systemic AA amyloidosis: epidemiology, diagnosis, and management. Clin Epidemiol 2014; 29: 369-77.

13 Çetinkaya E, Özgül MA, Kırkıl G, Özgül G, Acat M, Kamiloğlu E et al. A Case of Tracheobronchial Amyloidosis Treated with Endobronchial Therapy. Turkish Thoracic J 2013; 14: 39-42.

14. Fiorelli A, Accardo M, Galluccio G, Santini M. Tracheobronchial amyloidosis treated by endobronchial laser resection and self expanding $\mathrm{Y}$ stent. Arch Bronconeumol 2013; 49: 303-5. 